\title{
ENFERMEIROS DOS CENTROS DE ATENÇÃO PSICOSSOCIAL EM ÁLCOOL E DROGAS (CAPS AD): A FORMAÇÃO E A BUSCA PELO CONHECIMENTO ESPECÍFICO DA ÁREA
}

\author{
Divane de Vargas ${ }^{1}$, Fernando Augusto Bicudo Duarte ${ }^{2}$
}

\footnotetext{
${ }^{1}$ Doutor em Enfermagem Psiquiátrica. Professor Doutor do Departamento de Enfermagem Materno-Infantil e Psiquiátrica da Escola de Enfermagem da Universidade de São Paulo. São Paulo, Brasil. E-mail: vargas@.usp.br

${ }^{2}$ Enfermeiro. Bolsista PIBIC/CNPq 2007-2008. Enfermeiro Trainee do Hospital São Luis. São Paulo, Brasil. E-mail: fernando. augusto.duarte@usp.br
}

RESUMO: Este estudo objetivou identificar a formação em dependência química e as fontes de conhecimento utilizadas pelos enfermeiros que atuam nos Centros de Atenção Psicossocial em Álcool e Drogas. Os sujeitos foram os 16 enfermeiros que trabalhavam nos Centros de Atenção Psicossocial em álcool e outras drogas da cidade de São Paulo, entre setembro e dezembro de 2007. Os dados foram coletados pelos registros autogravados e submetidos à análise temática de conteúdo. Os resultados evidenciaram que $68,8 \%$ dos entrevistados não possuiam formação específica em álcool e outras drogas e 93,8\% não receberam preparo nessa área durante a graduação em enfermagem. As leituras em livros e revistas, a internet e o convício com os demais profissionais da equipe foram identificados como fontes de conhecimento para esses enfermeiros. Concluiu-se que existe carência de formação específica na área das substâncias psicoativas e que na ausência de educação formal na área, os enfermeiros lançam mão de outras fontes, nem sempre confiáveis, na busca do conhecimento.

DESCRITORES: Serviços comunitários de saúde mental. Transtornos relacionados ao uso de substâncias. Enfermeiro. Conhecimento. Álcool.

\section{NURSES IN ALCOHOL AND DRUG COMMUNITY MENTAL HEALTH SERVICES: TRAINING AND QUEST FOR KNOWLEDGE IN THE FIELD}

\begin{abstract}
This study aimed to identify the training in chemical dependency and the sources of knowledge used by nurses who work in Psycho-Social Community Alcohol and Drug Centers in Brazil. The subjects were 16 nurses who worked in these centers in the city of São Paulo between September and December of 2007. Data was collected through self-recorded registries and then submitted to thematic content analysis. The results showed that $68.8 \%$ of respondents did not have specific training in alcohol and other drugs, while $93.8 \%$ received no preparation in this area from their undergraduate nursing degree program. The participants identified relevant literature and periodicals, information online, and living interaction with other team members as sources of knowledge. This study concludes there is a lack of specific training in the field of psychoactive substances in formal nursing education; as a result, nurses resort to other - not always reliable - sources in their pursuit of nursing knowledge.
\end{abstract}

DESCRIPTORS: Community mental health services. Substance-related disorders. Nurse. Knowledge. Alcohol.

\section{LOS ENFERMEROS DE LOS SERVICIOS DE SALUD MENTAL Y ABUSO DE ALCOHOL Y DROGAS: LA FORMACIÓN Y LA BÚSQUEDA DE CONOCIMIENTO ESPECIFICO EN EL ÁREA}

RESUMEN: Este estudio tuvo como objetivo identificar la formación en la dependencia química y las fuentes de conocimiento utilizadas por los enfermeros que trabajan en los servicios de salud mental y abuso de alcohol y drogas. Los sujetos del estudio fueron 13 enfermeros que trabajaban en el Centro de Atención Psicosocial en alcohol y drogas de la ciudad de São Paulo, entre septiembre y diciembre de 2007. Los datos fueron recolectados a través de encuestas auto-grabadas y sometidos al análisis de contenido temático. Los resultados mostraron que el $68,8 \%$ de los encuestados no tienen formación específica en alcohol y otras drogas y el $93,8 \%$ no recibió ninguna preparación en esta área durante su formación en enfermería. Se identificaron como fuentes de conocimiento para esos enfermeros: las lecturas de libros y revistas, Internet y la convivencia con otros miembros del equipo. Se concluye que existe una falta de formación específica en materia de sustancias psicotrópicas y que, en ausencia de educación formal en el área, los enfermeros recurren a otras fuentes, no siempre fiables, en la búsqueda del conocimiento.

DESCRIPTORES: Servicios comunitarios de salud mental. Trastornos relacionados con sustancias. Enfermeras. Conocimiento. Alcohol. 


\section{INTRODUÇÃO}

A assistência aos problemas relacionados ao álcool e outras drogas sempre esteve ligada à assistência psiquiátrica, marcada pela violação dos direitos humanos e pela má qualidade prestada ao usuário, na maioria das vezes, centrada no modelo hospitalocêntrico. ${ }^{1}$ Em novembro de 1990, a declaração de Caracas vinculou a atenção psiquiátrica à atenção primária em saúde, aos modelos alternativos centrados na comunidade, em suas redes sociais, deixando de priorizar o papel hegemônico e centralizador do hospital psiquiátrico, permitindo ao usuário a permanência em seu território. Sob a influência da Reforma Psiquiátrica no Brasil, o Ministério da Saúde, em 1992, pela Portaria $n^{\circ}$ $224 / 1992^{2}$ passou a financiar e normatizar novos serviços de saúde mental, regulamentando os Núcleos/Centros de Atenção Psicossocial (NAPSs/ CAPSs), que deviam priorizar o tratamento ambulatorial de caráter interdisciplinar.

Em 2002, pela Portaria GM/MS n $336^{3}$, os CAPSs foram redefinidos e passaram a se constituir nas seguintes modalidades de serviço: CAPS I, CAPS II e CAPS III, definidos por ordem crescente de porte, complexidade e abrangência populacional. Nesta portaria, foram instituídos os Centros de Atenção Psicossocial Álcool e outras Drogas, na modalidade II (CAPS ad II), caracterizado como um serviço de Atenção Psicossocial para atendimento de pacientes com transtornos decorrentes do uso e dependência de substâncias psicoativas. O serviço deve ser composto por uma equipe mínima da qual o profissional enfermeiro faz parte.

Apesar da inserção desses profissionais no atendimento aos indivíduos com problemas relacionados ao uso de substâncias psicoativas nos CAPS ad, na literatura, é evidenciado que estes nem sempre são preparados para essa atuação, revelando que existe pouca preocupação das instituições formadoras com a temática álcool e outras drogas durante a formação do enfermeiro, o que contribui para o despreparo desses profissionais, quando se deparam com essa problemática, em seus locais de trabalho. ${ }^{4-5}$

Pesquisa que avaliou os conhecimentos adquiridos sobre os problemas relacionados ao uso de álcool pelos enfermeiros de uma universidade pública brasileira, durante a formação educacional, mostrou a existência de deficiência na educação formal desses profissionais, pois, a maioria dos sujeitos demonstrou pouco ou nenhum conhecimento sobre o tema uso do álcool e suas consequências. ${ }^{6}$
Estudos realizados com enfermeiros de unidades básicas distritais de saúde evidenciaram carência de conhecimentos da problemática do álcool e do alcoolismo entre esses profissionais, ${ }^{7} \mathrm{o}$ que contribui para o não reconhecimento daqueles usuários que buscam atendimento nesses serviços, com queixas inespecíficas que podem estar associadas ao uso prejudicial ou à dependência alcoólica. ${ }^{8}$ Outra pesquisa realizada com enfermeiros de hospitais gerais constatou que esses profissionais acreditavam que o alcoolismo tratava-se de doença mental. Os autores consideraram que a pouca clareza sobre o quadro do alcoolismo estava associada ao fato de que os enfermeiros têm recebido pouca informação e treinamento específico sobre a temática, o que contribui para o pouco conhecimento. ${ }^{9}$

A literatura nacional vem evidenciando que os enfermeiros possuem reduzido conhecimento a respeito da problemática das substâncias psicoativas, $^{6-9}$ reflexo do pouco preparo recebido durante a formação, ${ }^{4-5}$ assim, considerando que o enfermeiro constitui a equipe mínima dos CAPS $\mathrm{ad}^{3}$ e que esses serviços vêm se consolidando em espaços recentes de prática desse profissional, o que ocasiona a escassez de investigações conduzidas nesses cenários. E considerando o resultado dos estudos anteriores relacionados a essa temática, julgou-se importante verificar como esse fenômeno apresenta-se entre os enfermeiros que atuam em serviços especializados em álcool e outras drogas. Acredita-se que os resultados observados são importantes à enfermagem, pois, ao diagnosticar a atual situação do conhecimento e da formação desses enfermeiros, as discussões poderão subsidiar essas questões, quer na formação do enfermeiro generalista, quer no preparo dos profissionais que deverão atuar nos espaços de atenção aos usuários de álcool e outras drogas, o que, em última análise, poderá reverter em uma assistência de enfermagem de melhor qualidade.

Este estudo tem como objetivo identificar a formação em dependência química e as fontes de conhecimento utilizadas por enfermeiros que atuam nos Centros de Atenção Psicossocial em Álcool e Drogas da cidade de São Paulo.

\section{METODOLOGIA}

Trata-se de um estudo exploratório, de cunho qualitativo. Os dados foram coletados por entrevistas semiestruturadas, com a seguinte questão norteadora: "comente sobre seu preparo para atuar com dependentes químicos". Os sujeitos do estudo foram 
todos os 16 enfermeiros, oriundos dos 13 Centros de Atenção Psicossocial em Àlcool e Drogas que estavam em funcionamento no Município de São Paulo, no período da coleta de dados, compreendido entre setembro a dezembro de 2007. Inicialmente, foi feito contato telefônico com cada um dos sujeitos, para o agendamento das entrevistas. Nesse momento, o pesquisador deu ciência do projeto de pesquisa, solicitando a cooperação dos mesmos, deixando claro que a participação no estudo era voluntária, o anonimato seria garantido e os resultados utilizados somente para fins científicos.

Todos os sujeitos convidados aceitaram participar voluntariamente do estudo, cientes de que sua identificação seria mantida em sigilo e, posteriormente, autorizaram a divulgação dos dados, por meio de Termo de Consentimento Livre e Esclarecido. A análise de dados seguiu os passos da Análise de Conteúdo. ${ }^{10}$ Utilizou-se a "análise temática", como técnica para tratamento do material. ${ }^{11}$ As entrevistas foram gravadas, transcritas e lidas exaustivamente. Dessa forma, foram extraídos dos depoimentos os temas emergentes do discurso dos entrevistados. Estes foram agrupados em categorias temáticas, compatíveis com o método utilizado. As unidades de registro foram classificadas e agregadas em duas categorias de análise: O preparo para atuar com o dependente químico e à dependência e A busca pelo conhecimento específico para atuação no CAPS ad.

Com o objetivo de garantir os aspectos éticos no desenvolvimento da pesquisa, sua realização foi aprovada pelo Comitê de Ética em Pesquisa com seres humanos da Secretaria Municipal de Saúde de São Paulo, sob n ${ }^{\circ}$ 149/08.

\section{RESULTADOS}

Inicialmente serão apresentados os resultados referentes à categorização sociodemográfica dos participantes e a formação em saúde mental e em álcool e outras drogas. Na sequência, apresentam-se os resultados observados na análise qualitativa do dados.

\section{Caracterização sociodemográfica dos sujeitos}

Os sujeitos do estudo caracterizaram-se como indivíduos predominantemente do sexo feminino $(68,8 \%)$, na faixa etária média de 48 anos e formados há mais de 25 anos (43,8\%), em faculdades privadas $(68,8 \%)$. Dos entrevistados, $68,8 \%$ atuavam no CAPS ad há menos de quatro anos, e metade dos sujeitos (50\%) afirmou já ter trabalhado em algum serviço de psiquiatria, antes do CAPS ad. Aproximadamente 56,3\% dos enfermeiros informaram manter outro vínculo empregatício, além do CAPS ad.

\section{Caracterização quanto à formação em saúde mental e em álcool e outras drogas}

A maioria (75\%) dos sujeitos informou não possuir especialização em saúde mental. O mesmo ocorreu com a formação específica em álcool e outras drogas $(68,8 \%)$. Com relação à participação em cursos de capacitação, a maioria $(87,5 \%)$ informou ter feito algum curso desse tipo nos últimos cinco anos. Quando se analisou a formação, na graduação em enfermagem, de questões relacionadas às substâncias psicoativas, 93,8\% negaram ter recebido qualquer tipo de preparo na área.

A análise de conteúdo do material coletado nas entrevistas com os enfermeiros, possibilitou a criação de duas categorias, que são apresentadas a seguir.

\section{O preparo para atuar com o dependente químico e a dependência}

Esta categoria é constituída pelas seguintes temáticas: Temática I - O preparo formal durante a graduação para atuar com o dependente químico e a dependência; Temática II - A carência de capacitação dos profissionais para atuação no CAPS ad; Temática III - A importância da formação específica para atuar na área.

\section{Temática I - O preparo formal, durante a gra- duação, para atuar com o dependente químico e a dependência}

No que se refere ao preparo formal dos enfermeiros, na graduação, para atuar com as dependências químicas e o dependente, a análise das falas dos sujeitos foi consistente com os dados levantados sobre o perfil dessa população, que evidenciou que, do total dos sujeitos do estudo, a grande maioria informou não ter recebido tal preparo no curso de enfermagem. Quando se referiam ao preparo recebido na formação, os sujeitos citaram que muito pouco foi dado sobre álcool e drogas, sendo o conteúdo ministrado de modo rápido, algo impregnado de certo preconceito, o que, consequentemente, fez com que o assunto fosse tratado como um tabu.

Na minha graduação, eu lembro que eu tive esse assunto em psiquiatria, mas muito pouco (E.3). Quando eu fiz a faculdade, eu não tive preparo nenhum, nenhum. 
Era um assunto meio tabu, foi passado muito por cima, então, eu não tinha noção da extensão dessa problemática (E.6). Na faculdade, você tem um certo preparo pra lidar com um paciente no pronto-socorro, na UTI, clínica médica, só que o paciente com dependência química não tem preparo nenhum. Quando você sai da faculdade, sai com um preconceito muito grande (E.9). A minha formação na faculdade foi mínimo o ensino sobre essa questão (E.14).

\section{Temática II - A carência de capacitação dos profissionais para atuação no CAPS ad}

Aliado ao pouco preparo recebido durante a graduação, para atuação com o dependente químico, os enfermeiros do estudo mencionaram também que existe carência de iniciativas do próprio serviço no oferecimento de capacitações e treinamentos que subsidiem sua atuação.

Eu não tive formação na área e quando vim trabalhar aqui não me ofereceram nenhum curso de capacitação (E.3). Falta capacitação. Quando a gente vem trabalhar aqui não está preparado. Eles te jogam, vai lá e se vira, evocê acaba aprendendo assim aqui (E.6). Eu acho que apesar de lidar com o paciente de álcool e drogas ser algo muito sério, existe carência de treinamentos pra gente aqui no serviço, nós não temos nenhum tipo de capacitação (E.9). Na prefeitura não existe nenhum curso de capacitação em álcool e drogas (E.13).

A carência de iniciativas do próprio serviço soma-se ao pouco incentivo por parte desses que não facilitam a participação do enfermeiro na busca de formação específica, uma vez que, ao optar por capacitar-se, necessita repor a carga horária utilizada para esse fim, conforme mencionou uma enfermeira que estava cursando a especialização: [...] para que eu possa fazer essa especialização, eu não serei liberada do trabalho, eu vou ter que pagar horas em que estiver estudando (E.13).

Talvez o pouco incentivo seja um fato que justifique a baixa procura do profissional enfermeiro por cursos de capacitação, mesmo quando estes são gratuitos, conforme evidencia a fala que segue: [...] quando eu fui procurar o curso de especialização, que é gratuito, fiquei desapontada, apesar do grande número de CAPS ad aqui em São Paulo. No processo seletivo para esse curso que estou fazendo, para todas as vagas tinha somente eu de enfermeira (E.7).

\section{Temática III - A importância da formação específica para atuar na área}

Ainda no que se refere ao preparo formal para atuar no CAPS ad alguns enfermeiros perce- bem que a formação geral ou em outras áreas não responde às necessidades de trabalho impostas no CAPS ad. Consideram a formação específica importante, visto que a atuação nesse cenário demanda conhecimentos específicos.

A minha formação é toda de saúde pública, eu fiz especialização nessa área e eu achava que dava pra suprir, afinal de contas, vê parte de saúde pública, mas agora os casos são tão complexos que não é só saúde pública, então já é uma complexidade maior. Eu acho que essa formação, voltada, específica pra álcool e drogas é muito importante (E.11). No começo, eu não via muito importância, assim, diferença, sabe! Eu não sentia tanta necessidade, porque o paciente que chegava pra gente, não era assim um caso tão complexo, agora eu percebo, com o passar do tempo, que uma formação específica ajuda muito (E.16).

\section{A busca pelo conhecimento para atuação no CAPS ad}

Ao considerar os resultados apresentados na categoria anterior, relativa ao preparo para atuar com a dependência química e o dependente, em que se observou pouco preparo formal do enfermeiro para atuação no CAPS ad, esta categoria propõe-se a apresentar os dados relacionados às estratégias utilizadas pelos enfermeiros na busca do conhecimento para atuação nessa área. Para isso, a segunda categoria de análise foi dividida nas seguintes temáticas: Temática I - As capacitações e leituras; Temática II - O conhecimento por meio da troca de experiências e o aprender fazendo; Temática III - O conhecimento adquirido por meio de outras experiências profissionais na área de enfermagem psiquiátrica e saúde mental.

\section{Temática I - As capacitações e leituras}

Conforme os enfermeiros entrevistados, dentre as estratégias utilizadas na busca pelo conhecimento para atuar no CAPS ad, as especializações e as capacitações oferecidas na área constituem estratégias das quais o enfermeiro lança mão para respaldar sua atuação, segundo evidenciam as falas a seguir: [...] fiz uma capacitação na USP, eu preciso desse preparo como enfermeira para trabalhar aqui (E.2). Fiz especialização na UNIFESP, em dependências, com o pessoal do PROAD (E.7).

$\mathrm{Na}$ ausência dos espaços formais, como as capacitações e especializações, os enfermeiros buscam obter informações e conhecimentos por meio de leituras de livros: [...] quando eu cheguei aqui, eu fui tentar ver aquelas coisas. Tinham aí 
apostilas e livros e fui lendo, né. Essa parte mais da dependência química mesmo, aí e fui lendo (E.3). Eu fui lendo alguns livros sobre a dependência química, fui pegando um pouco de cada coisa (E.8). Eu leio muito, eu leio bastante, textos e livros sobre dependências, sobre abstinência, no uso de cocaína, no uso de álcool, no crack e vou me preparando, assim (E.9). E, também, na rede mundial de computadores, a Internet: só aprendo mesmo pela Internet (E.1).

\section{Temática II - O conhecimento por meio da troca de experiências e o aprender fazendo}

Uma outra fonte de conhecimento mencionada pelos enfermeiros foi a própria convivência e a troca de experiência com os outros profissionais da equipe. Esse conhecimento é adquirido no dia a dia, no convívio com os demais profissionais.

Eu fui aprendendo mesmo, conversando com os outros técnicos que trabalham aqui (E.1). Eu tenho um grupo com as psicólogas, então eu fui aprendendo com elas, como é que funcionava (E.3). Eu não tenho muita experiência, eu vim pra cá e fui também aprendendo com os terapeutas (E.5). Eu fui vendo como cada profissional tratava, então eu coletei um pouquinho de cada coisa (E.8). Peguei dicas dos outros profissionais que trabalham aqui (E.9).

Além do convívio com os demais profissionais da equipe, o próprio fazer do enfermeiro no dia a dia e o contato com os pacientes também subsidiam a aquisição de conhecimentos para atuar no CAPS ad: [...] eu fui aprendendo mesmo, foi durante meu trabalho diário (E.3). Fui aprendendo mesmo na prática, foi muito autodidata (E.4). Por meio do contato com os usuários. Eu aprendo muito com eles na vivência com eles, na prática (E.10). Adquiri conhecimento e experiência no dia a dia, você aprende mesmo é na prática (E.12).

\section{Temática III - O conhecimento adquirido por meio de outras experiências profissionais na área de enfermagem psiquiátrica e saúde mental}

A experiência prévia e o conhecimento adquirido em serviços de psiquiatria e saúde mental aparecem como fatores que favorecem o trabalho e a atuação do enfermeiro no CAPS ad, pois, ter tido experiência prévia nessas áreas, leva o profissional a compreender melhor a dependência química. Por que como eu não tinha um preparo e depois de muitos anos trabalhando com saúde mental, acho que eu mesma fui descobrindo as necessidades, sem formação (E.4).
Bom, eu já trabalhei em psiquiatria, não diretamente, tá, no hospital, onde a gente atendia muitos pacientes em surto e tem relação à dependência, pacientes, é, psiquiátricos. Então, eu tenho esse preparo (E.12). O que valeu pra mim foi a minha saída da faculdade e já entrar na psiquiatria, então, eu já tinha uma certa experiência no ramo (E.14). No entanto, embora exista esse conhecimento prévio que favorece o trabalho do enfermeiro no CAPS ad, conforme revela a fala a seguir, existem diferenças entre atender um paciente psiquiátrico e um dependente químico, o que, em última análise, remete à necessidade de buscar formação específica para atuação na área da dependência química. Só que existe diferença entre um paciente psiquiátrico e um paciente que é dependente químico, ta! (E.12).

\section{DISCUSSÃO}

Apesar do aumento dos prejuízos sociais e das consequências negativas na saúde da população, ocasionados pelo abuso de álcool e outras drogas, poucos enfermeiros têm recebido educação formal para reconhecer a dependência química em seus pacientes, o que permitiria a esse profissional, dentre outros, providenciar os encaminhamentos necessários e intervenções efetivas frente a essa problemática. ${ }^{12-13} \mathrm{~A}$ pouca atenção dada à questão das substâncias psicoativas no currículo dos enfermeiros, apontada pela literatura, ${ }^{6-9,12-13}$ é consistente com os resultados deste estudo, que mostraram que a maioria dos enfermeiros atuantes nos CAPS ad do Município de São Paulo, recebeu pouco ou nenhum tipo de preparo formal na área das dependências durante a graduação. O estudo apontou ainda que, do total dos enfermeiros entrevistados, só um quarto informou possuir formação em saúde mental. O resultado evidencia um dissenso entre os pressupostos estabelecidos pela Portaria GM/MS n 336 que, ao estabelecer a equipe mínima, determina que o enfermeiro dessa equipe deve possuir especialização em saúde mental, o que pode refletir deficiências na articulação entre as políticas de saúde e a educação do país. ${ }^{3}$

A carência de preparo formal dos enfermeiros para o enfrentamento da problemática das substâncias psicoativas deve-se, conforme já mencionado, ao fato de que muitos cursos de graduação não tem oferecido conteúdos sobre a temática em qualidade e quantidade suficientes; ${ }^{4-9,12}$ problema que tem sido relatado também em outros países. ${ }^{14}$ Alia-se a isso, a pouca preocupação das instituições formadoras em oferecer cursos de especialização na área e, quando disponibilizados 
aos profissionais, ou são muito concorridos, ou não são acessíveis financeiramente ao enfermeiro. Em última análise, a questão pode levar ao desinteresse do profissional pela problemática, pois a falta de conteúdo ou dificuldade na detecção e a abordagem do problema nos pacientes com transtornos relacionados ao uso de sustâncias psicoativas podem levar o enfermeiro, além de apresentar atitudes negativas, ${ }^{9}$ a sentir-se despreparado ${ }^{7-8}$ e, consequentemente, mostrar-se desinteressado pela assistência dessa clientela. ${ }^{4-5}$ A esse respeito, autores vêm demonstrando que a dificuldade de articulação entre a teoria e a prática representa o maior empecilho para atuação do enfermeiro na área das substâncias psicoativas. ${ }^{15}$ Por outro lado, o preparo recebido pelos estudantes com estágio específico no setor de álcool e drogas parece fortalecer a capacidade do profissional atuar nessa área depois de formado. ${ }^{16}$

Embora apontem pouca ou nenhuma formação na área de álcool e outras drogas durante a formação, observou-se que existe pouco movimento dos enfermeiros para a busca de aperfeiçoamento em nível de pós-graduação ou cursos de capacitação. Esse desinteresse pela formação para atuação nos CAPS ad pode estar relacionado, além do fato da dificuldade financeira para seu custeio e a escassez de cursos dessa natureza, há também pouco incentivo recebido pelo enfermeiro dos próprios serviços, visto que os sujeitos do presente estudo mencionam que aqueles que se dispõem a buscar pela formação, precisam enfrentar uma dupla jornada, pois não é facilitada a saída do enfermeiro para essas atividades, já que precisam repor a carga horária utilizada para o serviço, o que desmotiva. O fato acarreta prejuízos à assistência, pois para atender à demanda de usuários de álcool e outras drogas é necessário, conforme apontaram os sujeitos da pesquisa, preparo técnico-científico. Uma solução para o problema poderia ser o oferecimento da capacitação em serviço, estratégia que, segundo os resultados do estudo, pouco tem sido explorada como alternativa para formação dos recursos humanos nos CAPS ad.

O pouco preparo específico do enfermeiro que atua nos CAPS ad assume maior dimensão quando se verifica que a maioria, após a Reforma Psiquiátrica, não se sente preparada para atuar em enfermagem psiquiátrica ou saúde mental ${ }^{11} \mathrm{e}$, consequentemente, no contexto das substâncias psicoativas, o que acaba comprometendo sua inserção nos novos dispositivos de atenção à saúde mental. Isto gera dificuldade entre os enfermeiros para re- conhecer seu trabalho, como capaz de auxiliar no tratamento dessa população. Isso ocorre em parte, por não se sentirem preparados para atuar na área ou por não compreenderem seu espaço de atuação entre os profissionais da equipe técnica. ${ }^{17-18}$

De acordo com os resultados deste estudo, os enfermeiros percebem a necessidade de receber formação específica para atuar nessa área do conhecimento, porque o profissional vivencia sua limitação, lidando diretamente com o dependente químico, percebendo, assim, ser preciso conhecer os conteúdos específicos sobre o cuidado de enfermagem. ${ }^{12}$

A troca de experiências, dentro do próprio serviço, entre os membros da equipe, foi mencionada como uma das fontes de conhecimento, pelos enfermeiros, um resultado que é consistente com estudo anterior, que encontrou que os profissionais compartilhavam seus conhecimentos e percebiam a necessidade de formação dentro do ambiente do trabalho. ${ }^{12}$ A experiência ou formação na área de enfermagem psiquiátrica apareceu como um facilitador para o trabalho do enfermeiro no CAPS ad, porém, segundo os entrevistados, ela não é suficiente para o enfrentamento da problemática relacionada às substâncias psicoativas. Assim, os enfermeiros apontam a necessidade de uma formação específica para atuação na área, resultado consistente com estudo prévio semelhante, ${ }^{12}$ no qual os enfermeiros reconheciam a necessidade de especialização na área de álcool e outras drogas para atuação com o dependente químico.

Na ausência de preparo formal para atuação com o dependente químico, os enfermeiros tendem a lançar mão de outras fontes de conhecimento. Dentre estas, podem ser citadas a rede mundial de computadores (Internet) e a leitura de livros sobre o tema. A busca do conhecimento na Internet, apesar de válida deve ser utilizada com cautela, uma vez que os conhecimentos disponibilizados nem sempre são pautados em conhecimento científico, podendo comprometer a qualidade da assistência prestada pelo enfermeiro. Esse resultado é consistente com outros estudos que evidenciaram que o conhecimento apresentado pelos estudantes de enfermagem pautava-se no senso comum e nas informações da grande mídia, deixando de apoiarse no conhecimento técnico-científico específico da área. ${ }^{4-5,16-17}$ Além da Internet, as outras fontes onde o enfermeiro busca o conhecimento são as atividades práticas, realizadas no cotidiano da unidade, e a troca de experiências com os demais profissionais da equipe técnica. Isso pode ser preocupante, so- 
bretudo quando se observa que o enfermeiro tem pouco conhecimento específico para atuar com o dependente químico. Desta forma, ao respaldar sua prática na prática de outros profissionais, o enfermeiro corre o risco de reproduzir modelos inadequados de assistência.

Embora o estudo tenha sido realizado com enfermeiros de um único município, o que pode ser considerado uma limitação, apresenta avanços no conhecimento sobre a temática, já que possibilita diagnosticar a formação dos enfermeiros que atuam nos CAPS ad do município brasileiro com o maior número de CAPS ad da federação. Dados disponibilizados pelo Ministério da Saúde apontam que até janeiro de 2009, estavam cadastrados 186 CAPS ad. Desses, a maior concentração, 46 (24,7\%), estava no Estado de São Paulo e, desse total, 15 (32\%) funcionavam na cidade de São Paulo.

\section{CONCLUSÃO}

O estudo apresentado traz avanços para a enfermagem, no campo das substâncias psicoativas, pois fornece dados sobre a busca do conhecimento do enfermeiro diante da ausência de capacitações formais na área, para atuação nesse novo cenário de práticas, representado pelos CAPS ad. Além disso, contribui para a construção do conhecimento sobre como vem se dando a inserção e o preparo do enfermeiro nesses espaços, constatando que se configuram em um vasto campo de pesquisa para a enfermagem.

No que se refere à formação e à busca pelo conhecimento dos enfermeiros do CAPS ad, o presente estudo evidenciou que a maior parte dos enfermeiros que atua nesses espaços no município de São Paulo carece de formação específica na área das substâncias psicoativas e não recebeu preparo adequado para atuação com o dependente químico durante a graduação em enfermagem. Na ausência desse conhecimento formal, o enfermeiro tem lançado mão de outras fontes de conhecimento, como as leituras e as informações divulgadas na grande mídia, em uma análise mais profunda pode comprometer a assistência a essa clientela.

Os resultados do estudo contribuem para a discussão da inclusão de conteúdos específicos na temática das substâncias psicoativas nos currículos dos profissionais de saúde, além de evidenciar a necessidade desses serviços disponibilizarem estratégias que visem a capacitar formalmente seus profissionais, garantindo uma assistência de qualidade baseada em princípios técnico-científicos.
Há menos de uma década, os CAPS ad foram regulamentados em todo território nacional, portanto, poucos estudos têm sido realizados sobre esses serviços e, em específico, na área da enfermagem. Frente a isso, sugere-se que mais estudos dessa natureza sejam realizados, abrangendo outras amostras de serviços, no sentido de ampliar os dados sobre essa situação em outras regiões do país.

\section{REFERENCIAS}

1. Ministério da Saúde (BR), Secretaria de Atenção à Saúde, Coordenação Nacional DST / AIDS. A política do Ministério da Saúde para a atenção integral a usuários de álcool e outras drogas. Brasília (DF): MS; 2003.

2. Ministério da Saúde (BR). Portaria $n^{\circ} 224 / 92$, de 29 de janeiro de 1992: dispõe sobre normas e diretrizes para os atendimentos hospitalares e extra hospitalares. Diário Oficial da União, Brasília, 30 de janeiro de 1992. Seção 1, p.1168-70.

3. Ministério da Saúde (BR). Portaria/GM n 336 de 19 de fevereiro de 2002. Legislação em Saúde Mental 1990 -2002. Diário Oficial da União, Brasília, 20 de fevereiro de 2002. $3^{\text {a }}$ ed. rev. e atual. Brasília: Ministério da Saúde, 2004. p.125-36.

4. Teixeira LG, Villar LMA. A formação do enfermeiro e o fenômeno das drogas no estado do Rio de Janeiro, Brasil: atitudes e crenças. Rev Latino-am Enfermagem. 2005; 13(Spe):872-9.

5. Carraro TE, Hussein RG, Luís MAV. A formação do enfermeiro e o fenômeno das drogas no sul do Brasil: atitudes e crenças dos estudantes de enfermagem sobre o cuidado. Rev Latino-am Enfermagem. 2005; 13(Spe):863-71.

6. Pillon, SC. Atitudes dos enfermeiros com relação ao alcoolismo: uma avaliação de conhecimentos. Rev Eletrônica Enferm. 2005 [acesso 2010 Fev 02]; 7(3):303-7. Disponível em http:/ / www.fen.ufg.br/ revista/revista7_3/ok/original_07.htm

7. Vargas D, Luis MAV. Alcohol, alcoholism and alcohol addicts: conceptions and attitudes of nurses from district basic health centers. Rev Latino-am Enfermagem. 2008; 16(Spe):543-50.

8. Vargas D, Luis MAV, Oliveira MAF. Atendimento do alcoolista em serviços de atenção primária a saúde: as percepções e as condutas do enfermeiro. Acta Paul Enfermagem. 2010. Jan-Fev; 23(1):543-50.

9. Vargas D, Labate RC. Atitude de enfermeiros de hospital geral frente ao uso do álcool e ao alcoolismo. Rev Bras Enferm. 2006 Jan-Fev; 59(1):47-5.

10. Bardin L. Análise de conteúdo. Lisboa (PT): Edições 70; 2007.

11. Minayo MCS. O desafio do conhecimento: pesquisa qualitativa em saúde. São Paulo (SP): Hucitec; 2004. 
12. Happel B, Taylor C. Drug and alcohol education for nurses: have we examined the whole problem? J Addict Nurs. 1999 Out-Dez; 11(4):180-5.

13. Reismam BL, Shrader RW. Effect of nurses' attitudes toward alcoholism on their referral rate for treatment. Occup Health Nurs. 1984 Set-Out; 32(5):273-5.

14. Hussein RG International perspectives the educational experiences and previous orientation of undergraduate nursing students in alcohol and drug: the english context. J Addict Nurs. 2007 Jan-Mar; 18(1):47-52.

15. Rassol GH, Luis MAV. Talking drug and alcohol misuse in Brazil: priorities and challenges for nurses. Inter Nurs Review. 2004 Dez; 51(4):201-7.
16. Rassol GH. The educational experiences and previus orientation of undergraduate nursing studentes in alcohol and drug: the english context. J Addict Nurs. 2007 Jan-Mar; 18(1):47-52.

17. Oliveira AGB, Alessi NB. O trabalho de enfermagem em saúde mental: contradições e potencialidades atuais. Rev Latino-am Enfermagem 2003 Mai-Jun; 11(3):330-40.

18. Rocha, RM. O enfermeiro na equipe interdisciplinar do Centro de Atenção Psicossocial e as possibilidades de cuidar. Texto Contexto Enferm. 2005 Jul-Set; 14(3):350-7. 